Behavioral/Systems/Cognitive

\title{
Cocaine Self-Administration Produces a Progressive Involvement of Limbic, Association, and Sensorimotor Striatal Domains
}

\author{
Linda J. Porrino, David Lyons, Hilary R. Smith, James B. Daunais, and Michael A. Nader \\ Center for the Neurobiological Investigation of Drug Abuse, Department of Physiology and Pharmacology, Wake Forest University School of Medicine, \\ Winston-Salem, North Carolina 27157
}

\begin{abstract}
The primate striatum is composed of limbic, cognitive, and sensorimotor functional domains. Although the effects of cocaine have generally been associated with the ventral striatum, or limbic domain, recent evidence in rodents suggests the involvement of the dorsal striatum (cognitive and sensorimotor domains) in cocaine self-administration. The goals of the present studies were to map the topography of the functional response to cocaine throughout the entire extent of the striatum of monkeys self-administering cocaine and determine whether this response is modified by chronic exposure to cocaine. Rhesus monkeys were trained to self-administer $0.3 \mathrm{mg} / \mathrm{kg}$ per injection cocaine for $5 \mathrm{~d}$ (initial stages; $n=4$ ) or $100 \mathrm{~d}$ (chronic stages; $n=4$ ) and compared with monkeys trained to respond under an identical schedule of food reinforcement $(n=6)$. Monkeys received 30 reinforcers per session, and metabolic mapping was conducted at the end of the 5th or 100th self-administration session. In the initial phases of cocaine exposure, self-administration significantly decreased functional activity in the ventral striatum, but only in very restricted portions of the dorsal striatum. With chronic cocaine self-administration, however, the effects of cocaine intensified and spread dorsally to include most aspects of both caudate and putamen. Early experiences with cocaine, then, involve mainly the limbic domain, an area that mediates motivational and affective functions. In contrast, as exposure to cocaine continues, the impact of cocaine impinges progressively on the processing of sensorimotor and cognitive information, as well as the affective and motivational information processed in the ventral striatum.
\end{abstract}

Key words: cocaine; dopamine; self-administration; striatum; metabolic mapping; primate

\section{Introduction}

Cocaine is one of the most reinforcing drugs of abuse known to date. Although it binds with relatively equal potencies to dopamine (DA), serotonin, and norepinephrine transporters (Ritz et al., 1987), the behavioral actions of cocaine have been attributed to its effects on dopaminergic systems (Ritz et al., 1987; Giros et al., 1996; Volkow et al., 1997), particularly the mesolimbic DA system. Although the effects of cocaine in the ventral striatum (Roberts et al., 1980; Wise, 1984; Koob and Bloom, 1988) have been well characterized in both humans and animal models, the actions of cocaine in the dorsal striatum, which has a higher DA content than the ventral striatum, have received far less attention. Recently, there has been new interest in the importance of the dorsal striatum and its potential role in cocaine abuse (Berke and Hyman, 2000; Everitt and Wolf, 2002; Ito et al., 2002), but most

Received Dec. 18, 2003; revised Feb. 16, 2004; accepted Feb. 18, 2004. We dedicate this work to Patricia Goldman-Rakic.

This work was supported by National Institutes of Health Grants DA09085 and DA06634 from the National Institute on Drug Abuse. We thank Tonya Moore, Susan Nader, Clifford Hubbard, and Jennifer Sandridge for assistance in conducting these experiments.

Correspondence should be addressed to Dr. Linda J. Porrino, Department of Physiology and Pharmacology, Wake Forest University School of Medicine, Medical Center Boulevard, Winston-Salem, NC 27157-1083. E-mail: Iporrino@wfubmc.edu.

D0I:10.1523/JNEUROSCI.5578-03.2004

Copyright $\odot 2004$ Society for Neuroscience $\quad 0270-6474 / 04 / 243554-09 \$ 15.00 / 0$ of these studies have concentrated on rodent models of cocaine self-administration.

The striatum of primates, both human and nonhuman, is far more extensive and more complex than that of rodents. Organizationally, it can be divided into functional domains on the basis of corticostriatal connectivity patterns (see Fig. 1): limbic domain, ventral striatum; association domain, central caudate and putamen; and sensorimotor domain, dorsolateral caudate and putamen (Selemon and Goldman-Rakic, 1985; Alexander and Crutcher, 1990; Haber and Fudge, 1997). Each domain receives a unique set of dopaminergic inputs from the ventral tegmental area and substantia nigra (VTA/SN) (Szabo, 1980; Haber et al., 2000). This heterogeneous pattern of dopaminergic innervation is accompanied in turn by differences in the regulation of DA in each domain (Bradberry et al., 2000; Cragg et al., 2000, 2002).

Differences in DA dynamics have been hypothesized to underlie the distinct actions of cocaine in dorsal and ventral striatum (Wu et al., 2001). Bradberry and colleagues (2000) have reported that cocaine administration produces larger increases in the concentrations of extracellular DA in ventral as compared with dorsal striatum of monkeys; however, this work involved a relatively restricted area of striatum, particularly in the rostralcaudal dimension. One purpose of the present studies, therefore, was to produce a detailed map of the topography of the functional response to cocaine across limbic, association, and sensorimotor 
domains of nonhuman primates. The $2-\left[{ }^{14} \mathrm{C}\right]$ deoxyglucose (2DG) method was used to measure rates of glucose utilization, an index of functional activity, across the entire extent of the dorsal and ventral striatum of monkeys self-administering cocaine.

Chronic exposure to cocaine produces cellular and molecular neuroadaptations in numerous brain systems, but these are perhaps best characterized in the striatum. Reports from this laboratory, for example, have shown that repeated exposure to cocaine self-administration results in significant alterations of the dopaminergic system within the striatum of nonhuman primates (Letchworth et al., 2001; Nader et al., 2002). It is unclear, however, how or whether these changes in striatal DA systems modify the functional response to cocaine. The second goal of these studies, therefore, was to determine whether the response to cocaine within the functional domains of the striatum is modified by chronic exposure to drug self-administration.

\section{Materials and Methods}

Subjects. Fourteen experimentally naive adult male rhesus monkeys ( $\mathrm{Ma}$ caca mulatta) weighing between 7.6 and $11.5 \mathrm{~kg}$ (mean $\pm \mathrm{SD} ; 9.5 \pm 1.04$ ) at the start of the study served as subjects. Monkeys were housed individually in stainless steel cages with water ad libitum; animals had physical and visual contact with each other. Their body weights were maintained at $\sim 90-95 \%$ of free-feeding weights by banana-flavored pellets earned during the experimental sessions and by supplemental feeding of Lab Diet Monkey Chow, provided no sooner than $30 \mathrm{~min}$ after the session. All procedures were performed in accordance with established practices as described in National Institutes of Health Guide for Care and Use of Laboratory Animals. In addition, all procedures were reviewed and approved by the Animal Care and Use Committee of Wake Forest University.

Behavioral apparatus. Cocaine self-administration and foodreinforced responding occurred in ventilated and sound-attenuated operant chambers $(1.5 \times 0.74 \times 0.76 \mathrm{~m}$; Med Associates, East Fairfield, VT) designed to accommodate a primate chair (Model R001, Primate Products, Redwood City, CA). The chamber contained an intelligence panel $(48 \times 69 \mathrm{~cm})$, which consisted of two retractable levers $(5 \mathrm{~cm}$ wide $)$ and three stimulus lights. The levers were positioned within easy reach of the monkey sitting in the primate chair. One gram of food pellets was delivered from a feeder located on the top of the chamber. A peristaltic infusion pump (7531-10, Cole-Parmer Co., Chicago, IL) was used to deliver drug injections at a rate of $\sim 1 \mathrm{ml} / 10 \mathrm{sec}$ to those animals selfadministering cocaine. Operation of the chambers and data acquisition were accomplished with a Power Macintosh computer system with an interface (Med Associates).

Surgical procedures. All monkeys, including controls, were surgically prepared, under sterile conditions, with indwelling intravenous catheters and vascular access ports (Model GPV, Access Technologies, Skokie, IL). Monkeys were anesthetized with a combination of ketamine $(15 \mathrm{mg} / \mathrm{kg}$, i.m.) and butorphanol ( $0.03 \mathrm{mg} / \mathrm{kg}, \mathrm{i} . \mathrm{m}$.), and an incision was made near the femoral vein. After blunt dissection and isolation of the vein, the proximal end of the catheter was inserted into the vein for a distance calculated to terminate in the inferior vena cava. The distal end of the catheter was threaded subcutaneously to an incision made slightly off the midline of the back. The vascular access port was placed within a pocket formed by blunt dissection near this incision. Monkeys were given 24-48 hr recovery times before returning to food-reinforced responding. Approximately $5 \mathrm{~d}$ before the terminal procedure, each monkey was implanted with a chronic indwelling catheter into the adjacent femoral artery for collection of timed arterial blood samples during the 2DG procedure. The surgical procedures were identical to those described for the venous catheters. For monkeys in the initial exposure groups (see below), this catheter was implanted at the same time as the venous catheter.

Self-administration procedures. Monkeys were initially trained to respond on one of two levers by reinforcing each response on the correct lever with a $1 \mathrm{gm}$ banana-flavored pellet. Over a period of $\sim 3$ weeks, the interval between availability of food pellets was gradually increased until a $3 \mathrm{~min}$ interval was achieved [i.e., fixed-interval $3 \mathrm{~min}$ schedule (FI 3 -min)]. Under the final schedule conditions, the first response on the lever after 3 min resulted in the delivery of a food pellet; sessions ended after 30 food presentations. At the end of each session, the response levers were retracted, houselights and stimulus lights were extinguished, and animals remained in the darkened chamber for $\sim 30$ min before they were returned to their home cages. All monkeys responded under the FI 3 -min schedule of food presentation for at least 20 sessions and until stable performance was obtained ( $\pm 20 \%$ of the mean for three consecutive sessions, with no trends in response rates). When food-maintained responding was stable, the feeder was unplugged, and the effects of extinction on responding were examined for five consecutive sessions, after which responding was reestablished and maintained by food presentation.

After baseline performance had been established, all monkeys were surgically prepared with venous catheters, as described above, and randomly assigned to one of three groups. One group of monkeys served as controls $(n=6)$ and continued to respond under the FI 3-min schedule of food presentation for a total of 5 or 100 sessions. The remaining eight monkeys were assigned to a cocaine self-administration group $(n=$ 4/group): (1) initial (5 sessions) exposure to cocaine self-administration ( $0.3 \mathrm{mg} / \mathrm{kg}$ per injection) or (2) chronic (100 sessions) exposure to cocaine self-administration $(0.3 \mathrm{mg} / \mathrm{kg}$ per injection). Because $0.3 \mathrm{mg} / \mathrm{kg}$ cocaine per injection was considered a high dose for previously cocainenaive monkeys, for most animals this dose was achieved within two sessions by first allowing the monkey to self-administer $0.1 \mathrm{mg} / \mathrm{kg} \mathrm{co-}$ caine. Food-maintained performance was allowed to stabilize after surgery ( $\sim 4-6 \mathrm{~d}$ ) before cocaine self-administration sessions were begun. Before each experimental session, the back of the animal was cleaned with $95 \%$ ethanol and betadine scrub, and a 22 gauge Huber Point Needle (Model PG20-125, Access Technologies) was inserted into the port leading to the venous catheter, connecting an infusion pump, containing the cocaine solution, to the catheter. Before the start of the session, the pump was operated for $\sim 3 \mathrm{sec}$, filling the port with the dose of cocaine that was available during the experimental session. At the end of each session, the port was filled with heparinized saline $(100 \mathrm{U} / \mathrm{ml})$ to help prevent clotting.

For all groups, responding was maintained under an FI 3-min schedule, and sessions ended after 30 reinforcer presentations. Similar to training, each animal remained in the darkened experimental chambers with levers retracted for $30 \mathrm{~min}$ after the final reinforcer was obtained. Daily experimental sessions, conducted at approximately the same time each day, continued for 5 or $100 \mathrm{~d}$. Immediately after the last infusion on the final session, the 2DG procedure was conducted.

Measurement of local cerebral glucose utilization. For these experiments, the animals' catheters exited through an opening in the rear of the chamber, allowing all infusions and sampling to be accomplished remotely with minimal disruption to the animal. The 2DG procedure was initiated at the end of the last session, 2 min into the timeout, by the infusion of an intravenous pulse of $75 \mu \mathrm{Ci} / \mathrm{kg}$ 2-deoxy-D- $\left[{ }^{14} \mathrm{C}\right]$ glucose (PerkinElmer Life Sciences, Boston, MA; specific activity 50-55 mCi/ mmol) followed by a flush of heparinized saline. Timed arterial blood samples $(0.25,0.5,0.75,1,2,5,7.5,10,15,25,35$, and $45 \mathrm{~min})$ were drawn thereafter at a schedule sufficient to define the time course of the arterial $2-\left[{ }^{14} \mathrm{C}\right]$ deoxyglucose and glucose concentrations. Arterial blood samples were centrifuged immediately. Plasma ${ }^{14} \mathrm{C}$ concentrations were determined by liquid scintillation spectrophotometry (Beckman Instruments, Fullerton, CA), and plasma glucose concentrations were assessed using a glucose analyzer (Beckman Instruments). Approximately $45 \mathrm{~min}$ after tracer injection, the animals were killed by an intravenous overdose of sodium pentobarbital $(100 \mathrm{mg} / \mathrm{kg})$. Brains were removed rapidly (postmortem interval of 10-15 min), blocked in three parts, frozen in isopentane $\left(-45^{\circ} \mathrm{C}\right)$, and stored at $-70^{\circ} \mathrm{C}$ until they were processed for autoradiography. Coronal sections (20 $\mu \mathrm{m}$ thick) were cut in a cryostat maintained at $-22^{\circ} \mathrm{C}$. Four of every 20 sections were thaw-mounted on glass coverslips, dried on a hot plate, and apposed to Kodak magnetic resonance-1 film (Rochester, NY) for 15-30 d, along with a set of 

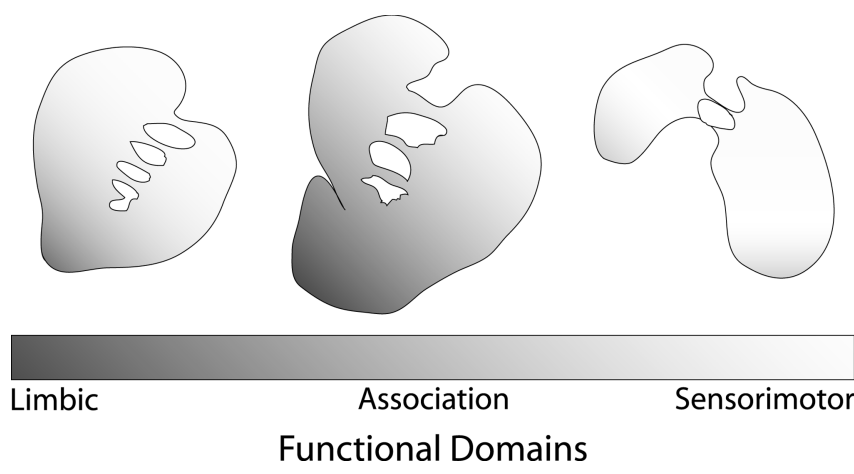

Figure 1. Schematic representation of functional domains within three representative levels of the striatum. The three levels represent the rostral precommissural striatum (level +24 ) where the shell of the accumbens is not present, the caudal precommissural striatum (level +20 ), and the postcommissural striatum (level +14 ). Limbic (dark gray), association (light gray), and sensorimotor (white) domains of the striatum are based on its cortical inputs, such that the ventral areas receive projections from limbic cortex and amygdala, the central division receives inputs from association areas of cortex, and the dorsal areas are innervated by sensory and motor related cortices. Adapted from Haber and McFarland (1999).

$\left[{ }^{14} \mathrm{C}\right]$ methylmethacrylate standards (Amersham Biosciences, Arlington Heights, IL) that had been calibrated previously for their equivalent ${ }^{14} \mathrm{C}$ concentration in $20 \mu \mathrm{m}$ brain sections. Autoradiograms were developed in Kodak GBX developer, indicator stop bath, and rapid fix at $68^{\circ} \mathrm{C}$.

Quantitative densitometry of autoradiograms was accomplished with a computer-assisted image-processing system (Imaging Research, St. Catharines, Ontario, Canada). Optical density measurements for each structure were made in a minimum of eight brain sections. Measurements were made bilaterally and averaged across hemispheres. Tissue ${ }^{14} \mathrm{C}$ concentrations were determined from the optical densities and a calibration curve obtained by densitometric analysis of the autoradiograms of the calibrated standards. Glucose utilization was then calculated using the operational equation of the method of Sokoloff et al. (1977), local-tissue ${ }^{14} \mathrm{C}$ concentrations, the time course of the plasma $2-\left[{ }^{14} \mathrm{C}\right]$ deoxyglucose and glucose concentrations, and the appropriate kinetic constants (Kennedy et al., 1978). Because of differences in the baseline levels of glycemia in some animals, the lumped constant was adjusted appropriate to the glucose levels according to procedures based on previous work (Kennedy et al., 1978; Schuier et al., 1990; Suda et al., 1990). Identification of brain structures was accomplished by comparison with adjacent thionin-stained sections.

The strategy for the analysis of rates of local cerebral glucose utilization in the striatum was adapted from the methods of Goldman-Rakic and colleagues (Levy et al., 1997) in which glucose utilization was measured in autoradiograms of the entire rostral-caudal and dorsal-ventral extent of the caudate. In the present study, rates of glucose utilization were measured in each functional domain (Fig. 1). Ten levels in the coronal plane (see Fig. 2) extending from the most rostral portions of the caudate and putamen $(+24)$ posterior to the most caudal levels of the putamen $(+8)$ were evaluated. At each level (where applicable), measurements were made in dorsal, central, and ventral portions of the putamen; dorsolateral, dorsomedial, central and ventral portions of the caudate; and the core and shell of the nucleus accumbens (see Fig. 3). Circular or elliptical tools were chosen to encompass as completely as possible the entire region of interest in both the left and right striatum. Tool size varied across regions and across levels, but the size of the analysis tool was kept constant for a given region and level across animals to ensure that equivalent portions of striatum were compared across groups. Care was taken, however, to eliminate artifacts such as holes or wrinkles that could bias the analysis inappropriately. A minimum of eight sections was chosen for analysis at each rostral-caudal level. Individual rates were averaged across sections to obtain a mean value for that region of interest at that level. All averages were weighted by the total number of pixels to prevent values of a small subset of pixels from biasing the data. Because no side-to-side differences were observed, values for right and left striatal regions were averaged as well. Furthermore, the SD of all pixels within



Figure 2. Schematic drawing depicting a lateral view of the striatum as it is situated within the entire rhesus monkey brain. The darker gray region represents the caudate, whereas the lighter gray region represents the putamen. Anterior-posterior levels at which rates of local cerebral glucose utilization were measured are represented by the vertical lines. Adapted from Levy et al. (1997).

each region was calculated. Whenever the SD was $>10 \%$ of the average, autoradiograms were reanalyzed to prevent errors. Figure 3 shows the locations of regions of interest at each striatal level. All autoradiograms were analyzed by two raters blind to the treatment of the animals. Interrater reliability was $96 \%$.

Statistical analysis. Standard statistics software (SPSS for Windows, Chicago, IL) was used for statistical analysis. Response rates maintained by food and cocaine are presented as the mean ( \pm SEM) for all monkeys in a group. Behavioral data were analyzed using repeated-measures ANOVA.

For global rates, cerebral metabolism was estimated in the caudate, putamen, and ventral striatum as the mean (weighted by region size) of all areas at all levels within each structure. Statistical analysis was performed in each subdivision of the caudate, putamen, and ventral striatum independently. Values of rates of local cerebral glucose utilization obtained for each individual region were analyzed by means of a two-way ANOVA for repeated measures (duration of cocaine exposure $\times$ rostralcaudal level, with rostral-caudal level as repeated measure). Post hoc Bonferroni $t$ tests for multiple comparisons compared rates of glucose utilization of groups self-administering cocaine with food-reinforced controls at each level.

\section{Results \\ Behavior}

The results of the behavioral aspects of these studies have been described in detail previously (Nader et al., 2002; Porrino et al., 2002). All monkeys were trained to respond under an FI 3-min schedule of food presentation. There were no differences in baseline response rates among the groups. After $\sim 3$ weeks of responding under this schedule, the pellet dispenser was unplugged, and responding was extinguished over five consecutive sessions, during which time response rates decreased in all monkeys. After extinction, food-maintained responding was reestablished, after which time each monkey was surgically prepared with indwelling catheters. Cocaine self-administration at 0.3 $\mathrm{mg} / \mathrm{kg}$ per injection was initiated in two of the groups. This was accomplished by substituting cocaine for food presentation. Selfadministration sessions continued for 5 or 100 daily sessions. For the control monkeys, responding continued to be maintained by food pellets.

Briefly, in the initial self-administration group, each monkey received the maximum number of injections per session (i.e., 30), which totaled $\sim 45 \mathrm{mg} / \mathrm{kg}$ cocaine over the course of the study. Re- 
Rostral Precommissural Striatum

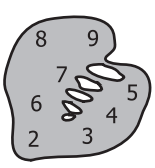
$+24$

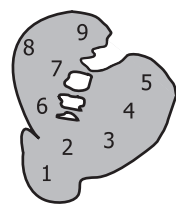

$+22$

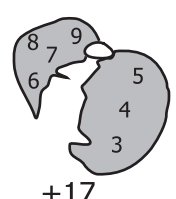



$+23$



$+20$



$+14$


$+11.5$

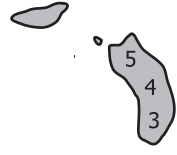

$+10$
Figure 3. Shown are schematic drawings representing the 10 anterior-posterior levels at which rates of glucose utilization were measured in each monkey extending from the rostral precommissural striatum, where the shell of the nucleus accumbens is not present, through the caudal precommissural striatum, where the shell is present, to the postcommissural striatum, where the nucleus accumbens is no longer evident. At each level the portions of the striatum (caudate, putamen, and accumbens) were subdivided further. The ventral striatum included the shell of the nucleus accumbens (1) and the rostral pole-core of the accumbens (2). The putamen was divided into ventral (3), central (4), and dorsal (5) portions. The caudate was divided into ventromedial (6), central (7), dorsomedial (8), and dorsolateral (9) portions.
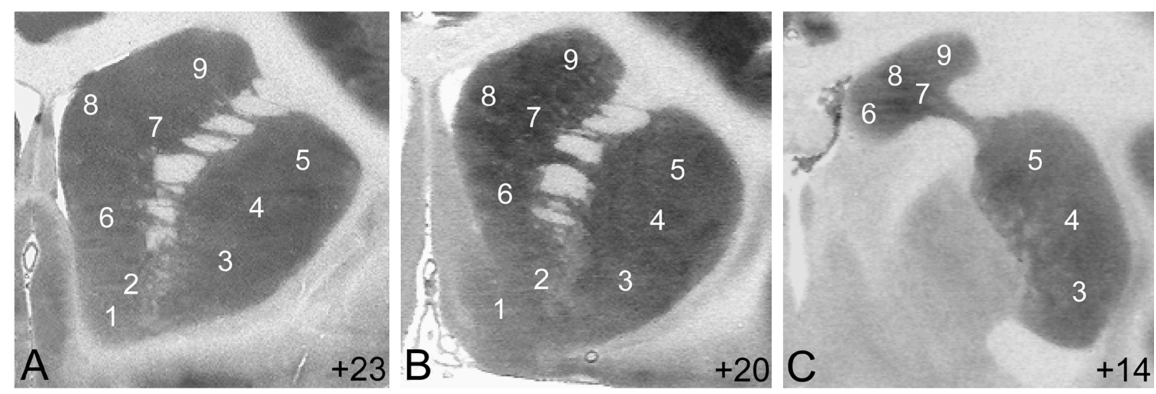

Figure 4. Autoradiograms from coronal sections through the rostral precommissural $(A)$, caudal precommissural $(B)$, and postcommissural $(C)$ striatum of a control monkey. The numbers represent the subdivisions of each region in which glucose utilization was measured: ventral striatum: shell of the nucleus accumbens (1) and rostral pole-core of the accumbens (2); putamen: ventral (3), central (4), and dorsal (5) regions; caudate: ventromedial (6), central (7), dorsomedial (8), and dorsolateral (9) portions.

sponse rates calculated across the last three sessions averaged $0.01 \pm$ 0.00 responses per second. For monkeys in the chronic selfadministration exposure group, the reinforcer remained constant for 100 consecutive sessions. Again each monkey received the maximum number of injections in each session over the course of the 100 daily sessions. Intake totaled $\sim 900 \mathrm{mg} / \mathrm{kg}$. Over the final three sessions, response rates in this group averaged $0.03 \pm 0.02$ responses per second.

\section{Local cerebral glucose utilization}

Patterns of rates of local cerebral glucose utilization in the striatum of normal control monkeys

Rates of glucose utilization were measured throughout the rostral-caudal extent of subdivisions of the caudate, putamen, and ventral striatum as shown in Figures 2-4. Figure 4 shows examples of autoradiograms of the striatum of a control monkey at three rostral-caudal levels. The main levels of analysis included the rostral precommissural striatum (Fig. $4 A$ ), where the nucleus accumbens is not clearly differentiated into distinct shell and core
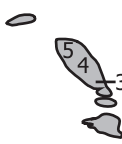

$+8$ subcompartments (levels +23 and +24 ); the caudal precommissural striatum (Fig. $4 B$ ), where the shell of the nucleus accumbens can be readily distinguished from the core of the nucleus accumbens (levels +19 , +20 , and +22 ); and the postcommissural striatum (Fig. 4C), which encompasses the striatum posterior to the anterior commissure (levels $+17,+14,+11.5,+10$, and +8 ), where the nucleus accumbens is absent.

In all monkeys, rates of glucose utilization were distributed heterogeneously within the striatum. In all controls monkeys, as reported previously (Palombo et al., 1990; Lyons et al., 1996; Porrino et al., 2002), there was a striking difference between rates of glucose utilization in the dorsal and ventral striatum (Fig. 4). Rates of glucose utilization were generally higher in the dorsal striatum, caudate $(56.2 \pm 1.6$ $\mu \mathrm{mol}$, mean $\pm \mathrm{SEM}$, per $100 \mathrm{gm} / \mathrm{min}$; areas 6-9), and putamen (56.3 $\pm 1.8 \mu \mathrm{mol}$, mean \pm SEM, per $100 \mathrm{gm} / \mathrm{min}$; areas 3-5) than in the ventral striatum, the nucleus accumbens, core, and shell (45.3 \pm 1.5 $\mu \mathrm{mol}$, mean $\pm \mathrm{SEM}$, per $100 \mathrm{gm} / \mathrm{min}$; areas 1-2).

Within the territories of the ventral striatum of control monkeys, in which responding was maintained by food, rates of glucose utilization were generally homogeneously distributed in the anterior-posterior plane (Fig. 5, Food Group) of both the shell and core of the nucleus accumbens. In contrast, rates of glucose utilization in the dorsal striatum of control monkeys were not uniform within the rostralcaudal dimension. In both the caudate (Fig. 6, Food Group) and the putamen (Fig. 7), the highest rates of glucose utilization were present in the vicinity of the anterior commissure (approximately the +19 level), with lower rates at both more rostral and caudal levels. This pattern was most readily distinguished in the most dorsal portions of both the caudate and putamen.

Effects of cocaine self-administration on patterns of local cerebral glucose utilization in the striatum

Cocaine self-administration decreased rates of glucose utilization across the three main subdivisions of the striatum considered in the present study. Global rates of cerebral metabolism were significantly reduced in ventral striatum $(F=13.81$; df $=2,11 ; p<$ $0.001)$, caudate $(F=10.04 ; \mathrm{df}=2,11 ; p<0.002)$, and putamen $(F=8.61 ; \mathrm{df}=2,11 ; p<0.006)$. These decreases were generally more intense and involved a wider regional extent in those monkeys that had self-administered for $100 \mathrm{~d}$ (chronic group) as compared with those who self-administered for only $5 \mathrm{~d}$ (initial group). Furthermore, in both initial and chronic groups, these effects were also regionally specific. The effects of selfadministered cocaine were greater in those portions of the striatum rostral to the anterior commissure than in the postcommissural striatum. 

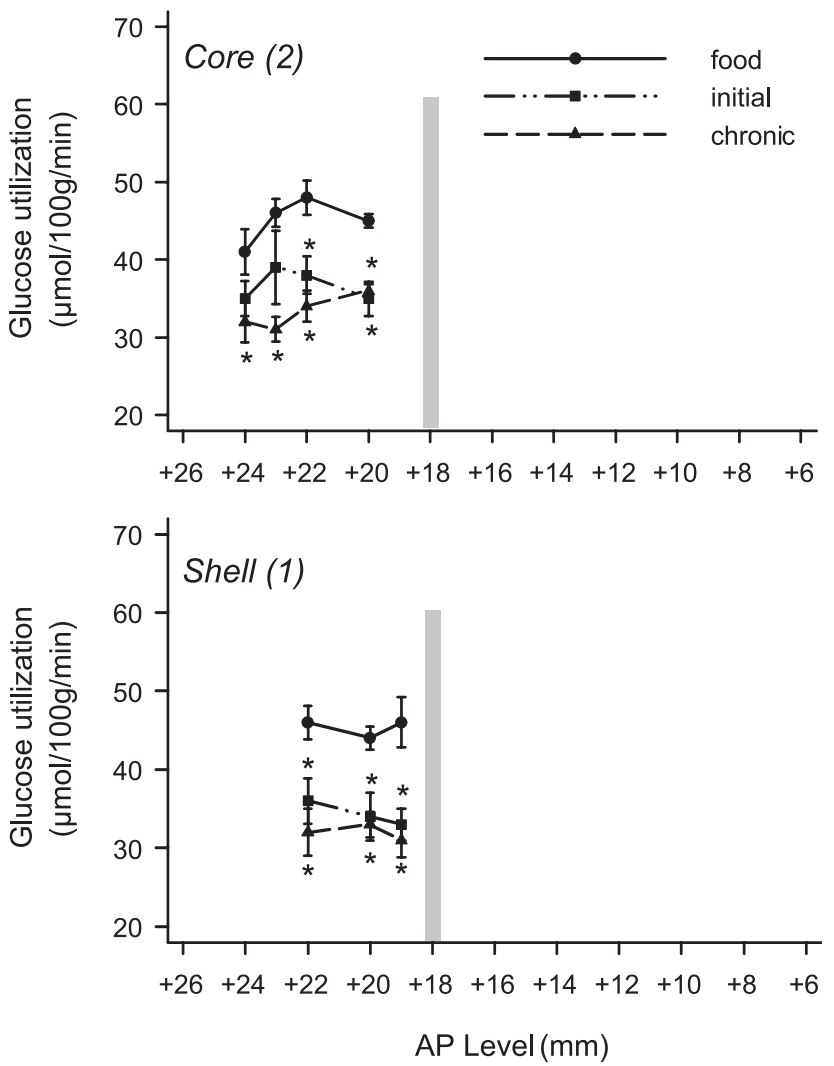

Figure 5. The effects of cocaine self-administration on rates of local cerebral glucose utilization across the anterior-posterior extent of the core (top) and shell (bottom) subdivisions of the ventral striatum of rhesus monkeys. Rates of glucose utilization of monkeys in the initial (5 d experience) and chronic (100 d experience) stages of self-administration are compared with rates of glucose utilization of control monkeys in which responding was maintained by food. Asterisks mark statistically significant differences $(p<0.05)$ from control. The vertical line in each panel represents the level of the anterior commissure. Note that glucose utilization is significantly reduced in both shell and core at both time points, but is reduced in the more rostral pole portion of the accumbens only at later time points.

\section{Ventral striatum}

In the initial stages (five sessions) of self-administration, rates of glucose utilization were significantly decreased $(-20$ to $-24 \%)$ across all three anterior-posterior levels of the shell (area 1) of the nucleus accumbens (levels: $+22,+20$, and +19 ) (Fig. 5, bottom panel). Glucose utilization was also significantly decreased $(-15$ to $-22 \%$ ) in the core (area 2) of the accumbens (levels: +22 and +20 ) (Fig. 5, top panel). There were no significant changes in the more rostral (area 2) portions of the core (levels: +24 and +23 ) (Fig. 5, top panel).

In the chronic group (100 sessions of cocaine selfadministration), cocaine also significantly reduced glucose utilization across all anterior-posterior levels of the shell (area 1) of the nucleus accumbens ( -26 to $-30 \%$ ) (Fig. 5 , bottom panel). In the core (area 2) of the accumbens (Fig. 5, top panel), however, rates of cerebral metabolism were now significantly decreased $(-20$ to $-33 \%)$ at all anterior-posterior levels from level +24 to +20 , including those areas that comprise the most rostral portions of the core (levels: +24 and +23 ).

Cocaine self-administration, then, resulted in large alterations in glucose utilization in the shell and core of the accumbens at both initial and chronic time points. Alterations in the more rostral portions of the core, however, were observed only after chronic self-administration.

\section{Dorsal striatum}

Cocaine self-administration also produced time-dependent reductions in rates of glucose utilization throughout both the caudate and putamen. There was a clear ventral to dorsal gradient in the magnitude of these decreases, with the most intense decreases present ventrally. Furthermore, alterations were confined to the precommissural dorsal striatum in the initial phases but spread to postcommissural striatum in the chronic phases of selfadministration (Figs. 6, 7).

In the initial phases (five sessions) of exposure to cocaine self-administration, glucose utilization was significantly reduced in the ventromedial (area 6; levels $+23,+22$, and +19 ), central (area 7; levels $+23,+22$, and +19 ), and dorsomedial (area 8; levels +23 and +22 ) portions of the caudate, but not in the dorsolateral (area 9) region (Fig. 6). Again, no significant differences as compared with control were evident in any portion of the postcommissural caudate at this time point.

With chronic exposure to self-administration (100 sessions), the effects of cocaine were generally more intense and more widespread, now including territories both rostral and caudal to those affected in the initial stages of self-administration (Fig. 6). Specifically, in the chronic stages of self-administration, glucose utilization was significantly reduced in the ventromedial (area 6; levels $+24,+23,+22,+20,+19,+17$, and +14$)$, central (area 7 ; levels +23 , +22 , and +19 ), dorsomedial (area 8; levels +24 , $+23,+22,+20,+19,+17$, and +14 ), and dorsolateral (area 9; levels +23 , +22 , and +19 ) portions of the caudate. These changes ranged from -18 to $-26 \%$ as compared with -12 to $-22 \%$ in the initial phase.

A similar pattern of metabolic alterations was found in the putamen, with the largest alterations in cerebral metabolism evident ventrally and increasing in magnitude and territory with longer exposure to self-administration (Fig. 7). Specifically, in the initial stages (five sessions) of self-administration, glucose utilization was significantly reduced in the ventral (area 3; levels $+23,+22$, and +19 ), central (area 4 ; levels $+23,+22$, and +20 ), and dorsal (area 5; levels $+23,+22$, and +19 ) portions of the putamen. Paralleling the patterns of glucose utilization in the caudate, no significant differences in rates of glucose utilization were evident in any portion of the putamen caudal to the anterior commissure at this time point.

With chronic exposure to self-administration (100 sessions), the effects of cocaine became more intense and more widespread with the involvement of territories both rostral and caudal to those affected in the initial stages of self-administration (Fig. 7). Specifically, in the chronic stages of self-administration, glucose utilization was significantly reduced in the ventral (area 3; levels $+24,+23,+22,+20,+19,+17,+14$, and +10$)$, central (area 4 ; levels $+23,+22,+20,+19$, and +17 ), and dorsal (area 5; levels $+23,+22$, and +19$)$ regions. These changes ranged from -17 to $-29 \%$ as compared with -14 to $-24 \%$ in the initial phase.

Cocaine self-administration, then, resulted in alterations in glucose utilization in the precommissural caudate and putamen at both initial and chronic time points. Alterations in the postcommissural portions of the dorsal striatum, however, were observed only after chronic self-administration.

\section{Discussion}

The present data demonstrate that cocaine self-administration alters functional activity, as reflected by rates of local cerebral glucose utilization, in broad expanses of both dorsal and ventral striatum. In the initial phases of cocaine exposure, a time chosen to model initial drug experimentation, cocaine produced signif- 

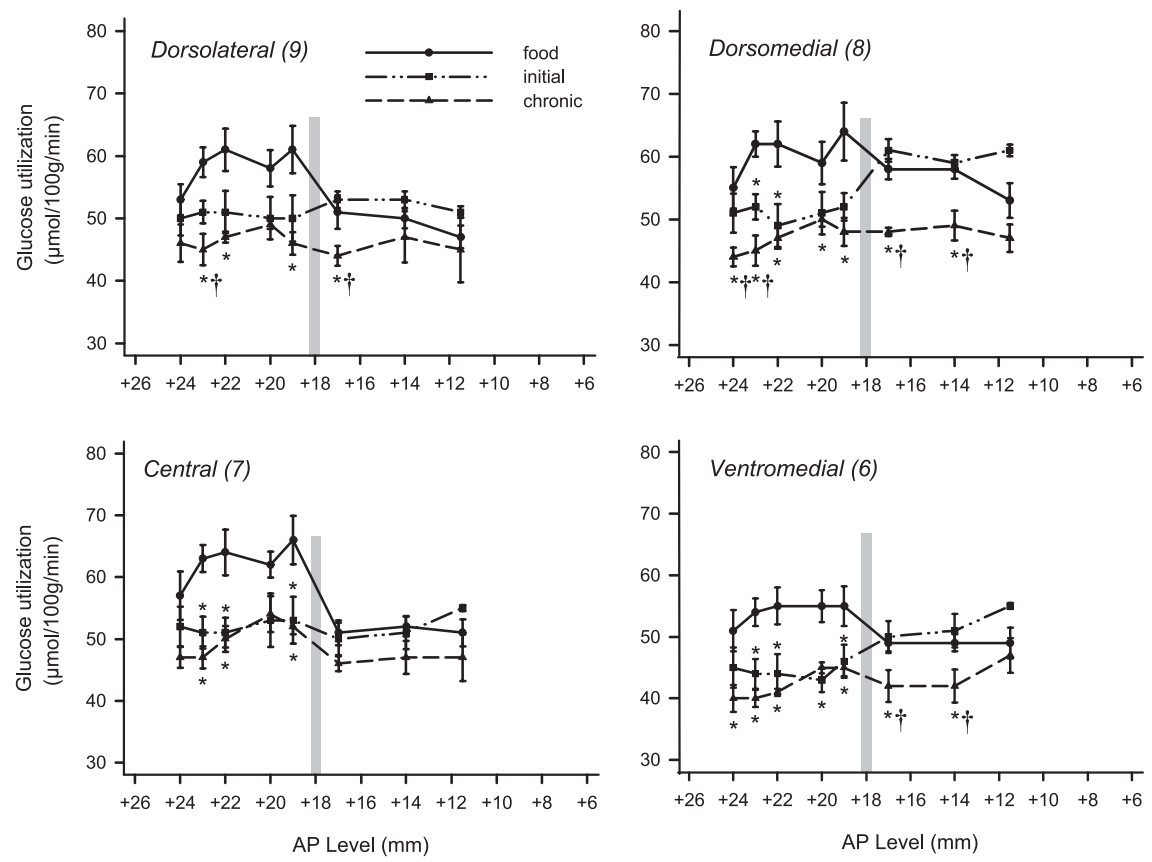

Figure 6. The effects of cocaine self-administration on rates of local cerebral glucose utilization across the anterior-posterior extent of the dorsolateral (top left), dorsomedial (top right), central (bottom left), and ventromedial (bottom right) subdivisions of the caudate of rhesus monkeys. Rates of glucose utilization of monkeys in the initial ( $5 \mathrm{~d}$ experience) and chronic ( $100 \mathrm{~d}$ experience) stages of self-administration are compared with rates of glucose utilization of control monkeys in which responding was maintained by food. Asterisks mark statistically significant differences $(p<0.05)$ from control. Daggers $(\dagger)$ mark statistically significant differences $(p<0.05)$ from initial stage. The vertical line in each panel represents the level of the anterior commissure. Note that glucose utilization is only significantly reduced in the postcommissural caudate at the later time points. Additionally, glucose utilization in the rostral-most levels of the caudate is also affected solely at the later time point.

icant decreases in functional activity in all portions of the ventral striatum, as well as in restricted portions of the dorsal striatum. The effects in the dorsal striatum at this time were confined to the caudal precommissural striatum, where the shell of the nucleus accumbens is most clearly defined (Fig. 8). With longer exposure, however, the effects of cocaine intensified and spread rostrally into the most anterior portions of striatum, caudally into postcommissural striatum, and dorsally to include the most dorsal aspects of both caudate and putamen (Fig. 8). Early experiences with cocaine, then, involve mainly limbic domains within the striatum, areas that mediate motivational and affective functions. In contrast, continued exposure to the effects of cocaine produces an expanded area of altered functional activity, encompassing association and sensorimotor domains, thereby influencing the substrates of cognitive, sensory, and motor processes.

\section{Role of the dorsal striatum}

Although the involvement of the dorsal striatum in the effects of noncontingent cocaine has been reported (Di Chiara and Imperato, 1988; Daunais and McGinty, 1994), its role in selfadministration has been recognized only recently (Bradberry, 2000; Ito et al., 2002). In rodents, significant increases in the concentration of extracellular DA have been reported to accompany both cocaine-seeking behavior and the contingent presentation of cocaine-associated stimuli (Ito et al., 2002). In monkeys, dopamine efflux is elevated in the ventromedial and central portions of the caudate during cocaine self-administration (Bradberry, 2000). The common element in these studies appears to be the extent of self-administration experience. Increased DA in the dorsal striatum reported by Ito et al. (2002) was found in rats responding under schedules of reinforcement that required ex- tensive training, whereas the concentrations of DA in more dorsal portions of the primate striatum were augmented after increased self-administration experience. The present data confirm and extend these findings by demonstrating the onset of the involvement of the dorsal striatum as cocaine exposure expands from the time of acquisition to the development of more chronic established drug-seeking behavior.

Although the dorsal striatum plays an integral role in the response to cocaine, the functional alterations in the ventral striatum were larger and more intense. This is consistent with studies that have shown that cocaine and amphetamine produce larger increases in extracellular DA concentrations in the ventral as compared with dorsal striatum of rodents (Carboni et al., 1989; Cass et al., 1992; Wu et al., 2002), monkeys (Bradberry, 2000; Bradberry et al., 2000), or humans (Drevets et al., 2001; Martinez et al., 2003). The extent of functional changes appears to reflect the degree of increase in the concentration of extracellular DA. Recent studies (Bradberry, 2000; Bradberry et al., 2000) have shown that cocaine self-administration produces greater elevations in DA levels in the ventral striatum, with the magnitude of increase diminishing dorsally. The preferential effects of cocaine in the ventral striatum have been hypothesized to derive from the differences in the rates of DA release and uptake in dorsal and ventral striatum of rodents (Wu et al., 2002). In addition, the distinct functional domains within the primate striatum can be distinguished on the basis of variation in DA release and uptake, resulting in differences in net DA availability (Cragg et al., 2002). Although the effects of cocaine were not examined, the distinctions in DA dynamics among functional domains is likely to be the source of the differential response to cocaine in the ventral striatum observed by Bradberry (2000) and in the present study.

If, as shown here, the most intense effects of cocaine are within ventral striatum, then what mechanisms are responsible for the significant changes in functional activity in more dorsal portions of the striatum? Haber and colleagues (2000) have recently described the organization of striatal connections with ventral midbrain dopamine cells and a schema for information integration across striatal regions. In their model, each striatal region is reciprocally connected with the VTA/SN. More specifically, the shell projects to portions of the VTA/SN that, in turn, project back to shell but also innervate the adjacent core. In turn, the core projects to portions of VTA/SN that then send afferents to both the core and the adjacent ventral caudate and putamen and so forth, thus creating a pattern of overlapping feed-forward projections from ventral to dorsal striatum. This circuitry provides an anatomical framework whereby the effects of cocaine in the ventral striatum can be transferred to association and sensorimotor domains in the dorsal striatum. Given the absence of a representation of the nucleus accumbens in the postcommissural striatum, this circuitry also provides an explanation of why, at least initially, self-administration does not alter functional activity in the more posterior parts of the striatum. The pattern of alter- 

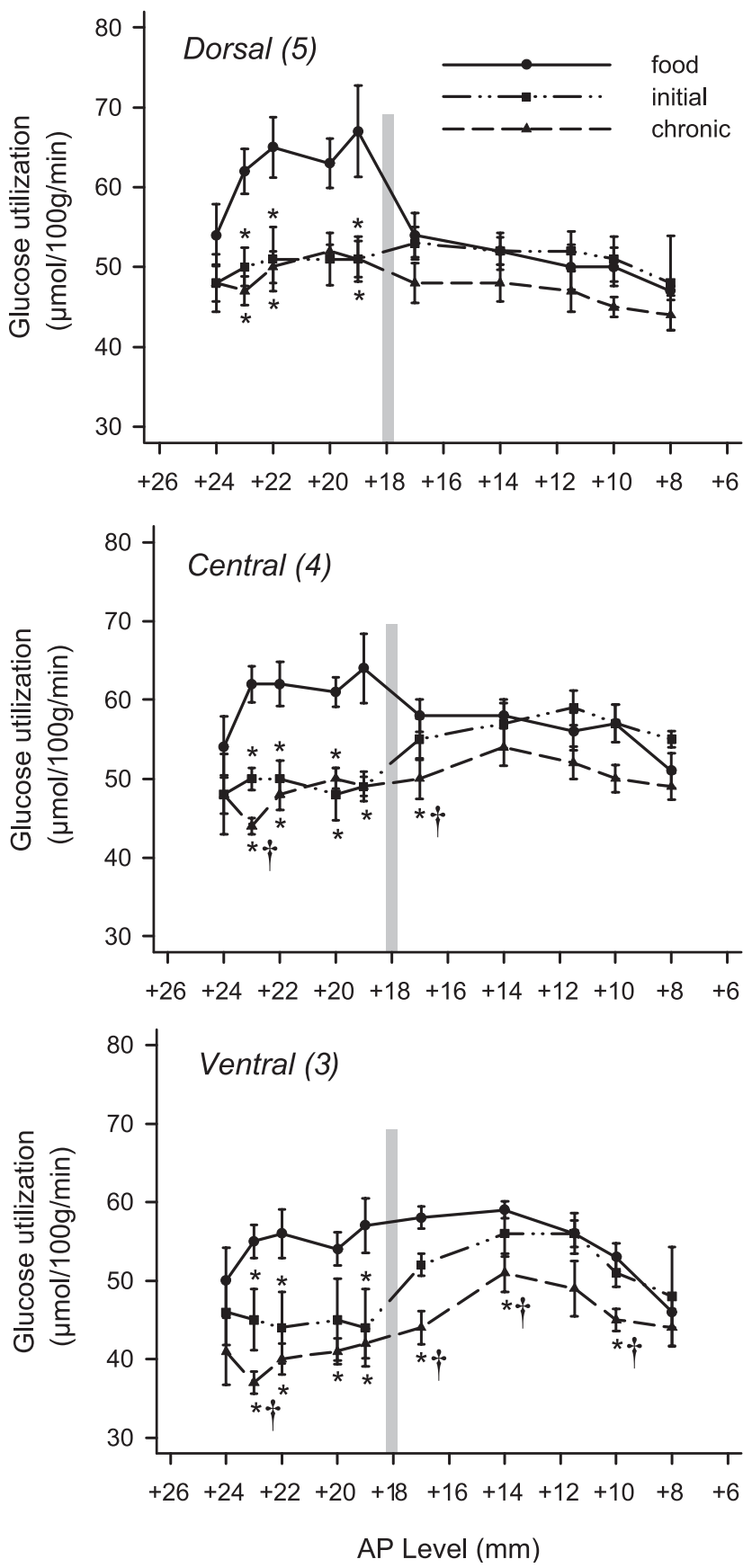

Figure 7. The effects of cocaine self-administration on rates of local cerebral glucose utilization across the anterior-posterior extent of the dorsal (top), central (center), and ventral (bottom) subdivisions of the putamen of rhesus monkeys. Rates of glucose utilization of monkeys in the initial ( $5 \mathrm{~d}$ experience) and chronic ( $100 \mathrm{~d}$ experience) stages of self-administration are compared with rates of glucose utilization of control monkeys in which responding was maintained by food. Asterisks mark statistically significant differences $(p<0.05)$ from control. Daggers $(\dagger)$ mark statistically significant differences $(p<0.05)$ from initial stage. The vertical line in each panel represents the level of the anterior commissure. Note that glucose utilization is only significantly reduced in the postcommissural putamen at the later time points. Additionally, glucose utilization in the rostral-most levels of the putamen is also affected solely at the later time point.

ations in glucose utilization described here, therefore, follow established principles of anatomical organization and, in turn, provide evidence of the functional relevance of this spiraling connectivity from ventral to dorsal striatum as described by Haber et al. (2000).

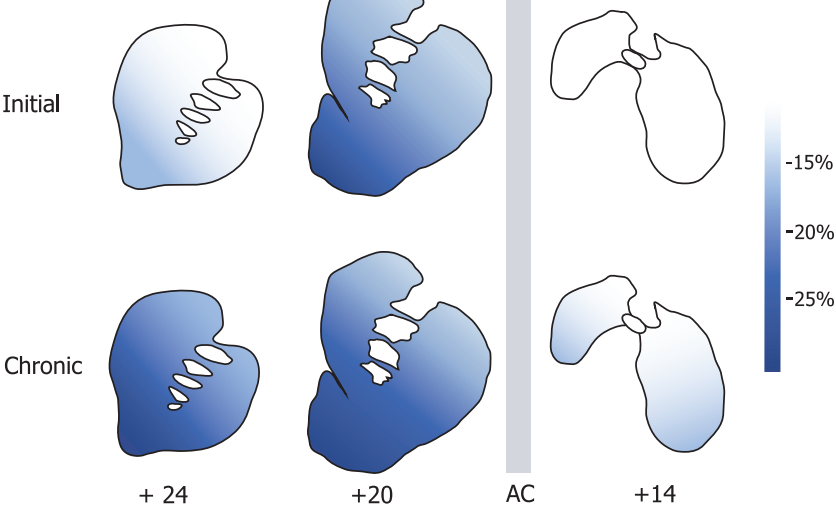

Figure 8. Schematic representation of the magnitude of the effects of cocaine at three levels of the striatum in the initial ( $5 \mathrm{~d}$ of exposure) and chronic ( $100 \mathrm{~d}$ of exposure) stages of cocaine self-administration. Shown are percentage changes from control, with the lighter blues indicating small changes and the darker blues denoting intense reductions in rates of glucose utilization as compared with rates in food controls. The scale at the right indicates the magnitude of change. The three levels represent the rostral precommissural striatum (level +24 ), where the shell of the accumbens is not present, the caudal precommissural striatum (level +20 ), and the postcommissural striatum (level +14 ). In the initial phases, changes are most intense in the caudal precommissural striatum (level +20 ), and no changes are observed posterior to the anterior commissure. After continued self-administration experience, the extent of striatum with altered functional activity expands to include both the more rostral precommissural regions and the striatum posterior to the anterior commissure. AC, Anterior commissure.

\section{Shift in the topography of the functional response to cocaine self-administration}

The dramatic shift in the pattern of alterations in glucose utilization that occurs with chronic exposure to cocaine selfadministration is consistent with reports of adaptations in dopamine and opioid systems. Prolonged cocaine self-administration in monkeys has been shown to result in an upregulation of dopamine transporter sites (Letchworth et al., 2001) and preprodynorphin mRNA (Fagergren et al., 2003), as well as a downregulation of dopamine $\mathrm{D}_{2}$ receptors (Moore et al., 1998; Nader et al., 2002). The pattern of these neuroadaptations followed an identical progression from ventral to dorsal striatum, as well as expanded rostrally and caudally with longer histories of cocaine self-administration. In these studies, however, the neuroadaptations developed over a longer time frame, with up to 2 years of exposure necessary for some changes. It appears that the functional changes in glucose utilization observed here precede the structural changes and therefore are not caused by changes in DA and opioid systems.

Although this shift in the topography of the striatal response to cocaine can be predicted by the anatomical organization of striatonigral-striatal projection patterns described by Haber and colleagues (2000), functional activity in the striatum may also be influenced by input from the cortex. Although in its initial stages cocaine self-administration affects only limited aspects of prefrontal cortex (Porrino and Lyons, 2000; Porrino et al., 2002), these effects may also widen in the cortex with continued exposure (Porrino and Lyons, 2000). Reciprocal and nonreciprocal corticothalamic connections (McFarland and Haber, 2002) may further amplify the impact of cocaine on the striatum. Regardless of their source, the broadening of the functional changes in the striatum that accompanies chronic cocaine self-administration develops in an orderly manner and follows established anatomical connectivity patterns.

Recently, the definition of the ventral striatum has been 
broadened to include the ventral putamen caudal to the anterior commissure (Fudge and Haber, 2002). Although functional activity was not altered in this area initially, with extended exposure to cocaine significant alterations in metabolism became clearly evident. If, like the more rostral ventral striatum, this portion of the ventral postcommissural putamen is also limbic in nature, it is not surprising that the most intense changes in the postcommissural striatum occurred in this region.

Recent conceptions of addiction have begun to focus on the compulsive nature of drug taking and the loss of control over drug-seeking that addicts frequently display (Koob and Le Moal, 1997; Berke and Hyman, 2000; Everitt et al., 2001). Drug use shifts from voluntary control in the initial stages to highly perseverative behavior patterns as drug use continues. Everitt and colleagues (Everitt et al., 2001; Ito et al., 2002) have suggested that this move is paralleled by a change in the dominant form of drug-associated behavioral processes, moving from goal-directed learning during acquisition to habit formation with increased time and exposure. Because the ventral striatum is thought to underlie goal-directed learning processes and the dorsal striatum is necessary for habit formation, the progression in the response to chronic cocaine reinforcement shown here, advancing from the ventral striatum to encompass the dorsal striatum, may provide an anatomical basis for this behavioral shift from voluntary drug use to addiction.

\section{Conclusions}

The present studies have shown that the functional consequences of cocaine are not restricted to the ventral striatum but can include large portions of the dorsal striatum as well. Moreover, as exposure to cocaine continues, the impact of cocaine progressively impinges on the processing of sensorimotor and cognitive information, as well as affective and motivational information processed in the ventral striatum. Questions clearly remain as to whether the altered response to cocaine persists or reverts to its earlier topography with abstinence, but perhaps more important is whether these changes alter the response to other stimuli or tasks that recruit sensory, motor, cognitive, or affective processes.

\section{References}

Alexander GE, Crutcher MD (1990) Functional architecture of basal ganglia circuits: neural substrates of parallel processing. Trends Neurosci 13:266-271.

Berke JD, Hyman SE (2000) Addiction, dopamine, and the molecular mechanisms of memory. Neuron 25:515-532.

Bradberry CW (2000) Acute and chronic dopamine dynamics in a nonhuman primate model of recreational cocaine use. J Neurosci 20:7109-7115.

Bradberry CW, Barrett-Larimore RL, Jatlow P, Rubino SR (2000) Impact of self-administered cocaine and cocaine cues on extracellular dopamine in mesolimbic and sensorimotor striatum in rhesus monkeys. J Neurosci 20:3874-3883.

Carboni E, Imperato A, Perezzani L, Di Chiara G (1989) Amphetamine, cocaine, phencyclidine and nomifensine increase extracellular dopamine concentrations preferentially in the nucleus accumbens of freely moving rats. Neuroscience 28:653-661.

Cass WA, Gerhardt GA, Mayfield RD, Curella P, Zahniser NR (1992) Differences in dopamine clearance and diffusion in rat striatum and nucleus accumbens following systemic cocaine administration. J Neurochem 59:259-266.

Cragg SJ, Hille CJ, Greenfield SA (2000) Dopamine release and uptake dynamics within nonhuman primate striatum in vitro. J Neurosci 20:8209-8217.

Cragg SJ, Hille CJ, Greenfield SA (2002) Functional domains in dorsal striatum of the nonhuman primate are defined by the dynamic behavior of dopamine. J Neurosci 22:5705-5712.

Daunais JB, McGinty JF (1994) Acute and chronic cocaine administration differentially alters striatal opioid and nuclear transcription factor $\mathrm{mR}$ NAs. Synapse 18:35-45.

Di Chiara G, Imperato A (1988) Drugs abused by humans preferentially increase synaptic dopamine concentrations in the mesolimbic system of freely moving rats. Proc Natl Acad Sci USA 85:5274-5278.

Drevets WC, Gautier C, Price JC, Kupfer DJ, Kinahan PE, Grace AA, Price JL, Mathis CA (2001) Amphetamine-induced dopamine release in human ventral striatum correlates with euphoria. Biol Psychiatry 49:81-96.

Everitt BJ, Wolf ME (2002) Psychomotor stimulant addiction: a neural systems perspective. J Neurosci 22:3312-3320.

Everitt BJ, Dickinson A, Robbins TW (2001) The neuropsychological basis of addictive behaviour. Brain Res Brain Res Rev 36:129-138.

Fagergren P, Smith HR, Daunais JB, Nader MA, Porrino LJ, Hurd YL (2003) Temporal upregulation of prodynorphin mRNA in the primate striatum after cocaine self-administration. Eur J Neurosci 17:2212-2218.

Fudge JL, Haber SN (2002) Defining the caudal ventral striatum in primates: cellular and histochemical features. J Neurosci 22:10078-10082.

Giros B, Jaber M, Jones SR, Wightman RM, Caron MG (1996) Hyperlocomotion and indifference to cocaine and amphetamine in mice lacking the dopamine transporter. Nature 379:606-612.

Haber SN, Fudge JL (1997) The primate substantia nigra and VTA: integrative circuitry and function. Crit Rev Neurobiol 11:323-342.

Haber SN, McFarland NR (1999) The concept of the ventral striatum in nonhuman primates. Ann NY Acad Sci 77:33-48.

Haber SN, Fudge JL, McFarland NR (2000) Striatonigrostriatal pathways in primates form an ascending spiral from the shell to the dorsolateral striatum. J Neurosci 20:2369-2382.

Ito R, Dalley JW, Robbins TW, Everitt BJ (2002) Dopamine release in the dorsal striatum during cocaine-seeking behavior under the control of a drug-associated cue. J Neurosci 22:6247-6253.

Kennedy C, Sakurada O, Shinohara M, Jehle J, Sokoloff L (1978) Local cerebral glucose utilization in the normal conscious macaque monkey. Ann Neurol 4:293-301.

Koob GF, Bloom FE (1988) Cellular and molecular mechanisms of drug dependence. Science 242:715-723.

Koob GF, Le Moal M (1997) Drug abuse: hedonic homeostatic dysregulation. Science 278:52-58.

Letchworth SR, Nader MA, Smith HR, Friedman DP, Porrino LJ (2001) Progression of changes in dopamine transporter binding site density as a result of cocaine self-administration in rhesus monkeys. J Neurosci 21:2799-2807.

Levy R, Friedman HR, Davachi L, Goldman-Rakic PS (1997) Differential activation of the caudate nucleus in primates performing spatial and nonspatial working memory tasks. J Neurosci 17:3870-3882.

Lyons D, Friedman DP, Nader MA, Porrino LJ (1996) Cocaine alters cerebral metabolism within the ventral striatum and limbic cortex of monkeys. J Neurosci 16:1230-1238.

Martinez D, Slifstein M, Broft A, Mawlawi O, Hwang DR, Huang Y, Cooper T, Kegeles L, Zarahn E, Abi-Dargham A, Haber SN, Laruelle M (2003) Imaging human mesolimbic dopamine transmission with positron emission tomography. Part II: Amphetamine-induced dopamine release in the functional subdivisions of the striatum. J Cereb Blood Flow Metab 23:285-300.

McFarland NR, Haber SN (2002) Thalamic relay nuclei of the basal ganglia form both reciprocal and nonreciprocal cortical connections, linking multiple frontal cortical areas. J Neurosci 22:8117-8132.

Moore RJ, Vinsant SL, Nader MA, Porrino LJ, Friedman DP (1998) Effect of cocaine self-administration on dopamine D2 receptors in rhesus monkeys. Synapse 30:88-96.

Nader MA, Daunais JB, Moore T, Nader SH, Moore RJ, Smith HR, Friedman DP, Porrino LJ (2002) Effects of cocaine self-administration on striatal dopamine systems in rhesus monkeys: initial and chronic exposure. Neuropsychopharmacology 27:35-46.

Palombo E, Porrino LJ, Bankiewicz KS, Crane AM, Sokoloff L, Kopin IJ (1990) Local cerebral glucose utilization in monkeys with hemiparkinsonism induced by intracarotid infusion of the neurotoxin MPTP. J Neurosci 10:860-869.

Porrino LJ, Lyons D (2000) Orbital and medial prefrontal cortex and psychostimulant abuse: studies in animal models. Cereb Cortex 10:326-333.

Porrino LJ, Lyons D, Miller MD, Smith HR, Friedman DP, Daunais JB, Nader MA (2002) Metabolic mapping of the effects of cocaine during the ini- 
tial phases of self-administration in the nonhuman primate. J Neurosci 22:7687-7694.

Ritz MC, Lamb RJ, Goldberg SR, Kuhar MJ (1987) Cocaine receptors on dopamine transporters are related to self-administration of cocaine. Science 237:1219-1223.

Roberts DC, Koob GF, Klonoff P, Fibiger HC (1980) Extinction and recovery of cocaine self-administration following 6-hydroxydopamine lesions of the nucleus accumbens. Pharmacol Biochem Behav 12:781-787.

Schuier F, Orzi F, Suda S, Lucignani G, Kennedy C, Sokoloff L (1990) Influence of plasma glucose concentration on lumped constant of the deoxyglucose method: effects of hyperglycemia in the rat. J Cereb Blood Flow Metab 10:765-773.

Selemon LD, Goldman-Rakic PS (1985) Longitudinal topography and interdigitation of corticostriatal projections in the rhesus monkey. J Neurosci 5:776-794.

Sokoloff L, Reivich M, Kennedy C, Des Rosiers MH, Patlak CS, Pettigrew KD, Sakurada O, Shinohara M (1977) The $\left[{ }^{14} \mathrm{C}\right]$ deoxyglucose method for the measurement of local cerebral glucose utilization: theory, procedure, and normal values in the conscious and anesthetized albino rat. J Neurochem 28:897-916.
Suda S, Shinohara M, Miyaoka M, Lucignani G, Kennedy C, Sokoloff L (1990) The lumped constant of the deoxyglucose method in hypoglycemia: effects of moderate hypoglycemia on local cerebral glucose utilization in the rat. J Cereb Blood Flow Metab 10:499-509.

Szabo J (1980) Organization of the ascending striatal afferents in monkeys. J Comp Neurol 189:307-321.

Volkow ND, Wang GJ, Fischman MW, Foltin RW, Fowler JS, Abumrad NN, Vitkun S, Logan J, Gatley SJ, Pappas N, Hitzemann R, Shea CE (1997) Relationship between subjective effects of cocaine and dopamine transporter occupancy. Nature 386:827-830.

Wise RA (1984) Neural mechanisms of the reinforcing action of cocaine. NIDA Res Monogr 50:15-33.

Wu Q, Reith ME, Kuhar MJ, Carroll FI, Garris PA (2001) Preferential increases in nucleus accumbens dopamine after systemic cocaine administration are caused by unique characteristics of dopamine neurotransmission. J Neurosci 21:6338-6347.

Wu Q, Reith ME, Walker QD, Kuhn CM, Carroll FI, Garris PA (2002) Concurrent autoreceptor-mediated control of dopamine release and uptake during neurotransmission: an in vivo voltammetric study. J Neurosci 22: $6272-6281$. 